\author{
Norman Fitz-Coy \\ Anindya Chatterjee \\ Department of Aerospace \\ Engineering, Mechanics, and \\ Engineering Science \\ University of Florida \\ Gainesville, FL 32611-2031
}

\title{
Actuator Placement in Multi-Degree-of-Freedom Vibration Simulators
}

\begin{abstract}
A method for comparing candidate actuator configurations for multi-degree-of-freedom (MDOF) vibration simulators is presented. The method has its roots in the comparison of achievable subspaces and maximum error bounds; the comparison is accomplished via a QR decomposition. In instances where two configurations yield the same error bound, the ratio of the largest to smallest singular value is used to determine the "best"' configuration. Both amplitude bounds at given sensor locations and relative significance of each sensor's output, if known, can be incorporated in the analysis. Through numerical examples, it is demonstrated that no simple rule of thumb criterion appears to exist for the selection of actuator placement in MDOF vibration simulators. (c) 1994 John Wiley \& Sons, Inc.
\end{abstract}

\section{INTRODUCTION}

Due to the changes in vibration test specifications, vibration test facilities must become more versatile and more powerful. The changes in test specifications are driven by the design and utilization of increasingly sophisticated equipment, and as such, it is expected that within a few years, multi-degree-of-freedom (MDOF) vibration simulators will become the norm rather than the aberration.

Specialized laboratories now require MDOF vibration simulators that handle a combination of both larger loads and higher frequency ranges than currently achievable. The combination of high load capacity and high frequency bandwidth results in one or more of the system's natural frequencies lying within the frequency range of the simulator's operation (Fitz-Coy, 1992; Schmidt, 1983; Raasch, 1983; Hahn and Raasch, 1986; Woyski and Tauscher, 1992). At or near resonance, the system's response tends to be dominated by the resonant mode and the pattern of the amplitudes of vibration at different points on the structure tends to be close to the resonant mode shape. Thus, it is difficult to get an arbitrary pattern of amplitudes at various specified points on the structure when the structure is excited at or near the resonant frequency.

For versatility, vibration simulators require that their design must work well not just for any one given load, but for a large variety of loads within some range. Consequently, in the design stage, there is no accurate model available for determining the optimal actuator locations-no such model exists. The designer is forced to work with a very crude model corresponding to some commonly encountered loading configuration.

The problem addressed in this article is that of actuator placement in MDOF vibration simulators. The need for MDOF vibration simulators that realistically reproduce the actual vibration environment has been acknowledged by vibration test engineers for some time (Fitz-Coy, 1992; Schmidt, 1983; Raasch, 1983; Hahn and 
Raasch, 1986; Woyski and Tauscher, 1992). This work was motivated by this need and the apparent lack of "concrete" solution methods.

To date, previous design approaches for MDOF vibration simulators (Raasch, 1983; Hahn and Raasch, 1986; Woyski and Tauscher, 1992) have been based on physical constraints and engineering judgement. In this article we present a criterion, which when supplemented with engineering judgement, will result in improved MDOF vibration simulators.

In the following sections, the problem of actuator placement, as it applies to MDOF vibration simulators, is described. A method for determining actuator placement is presented. The method is demonstrated with a numerical example. Finally, conclusions are presented.

The system considered is composed essentially of two components: the first component is the "table", on it is mounted the second component, the "load." There are $r$ actuators that are capable of exerting forces on $r$ given locations of the table. Sensors monitor the motion of $m$ specified locations on the load.

In the operation of a vibration simulator, the desired motion of the monitored points on the load will be specified. Typically, the actuators will usually be driven through displacement commands, reducing the problem to that of finding the input displacements at the $r$ actuator locations that will best approximate the desired responses at the $m$ monitored locations. The problem of choosing the $r$ actuator locations that will be most effective for the full range of displacement patterns, frequencies, and loading configu- rations is not trivial. In fact, it is not guaranteed that an arbitrary pattern of responses at the $m$ monitored locations will be exactly achievable with $r$ actuators, even for the case where $r>m$. The system shown in Fig. 1 can be used to demonstrate this point. Consider a scenario where $k_{10}=k_{11}=c_{10}=c_{11}=0$, sensors are located at masses $m_{6}$ through $m_{8}(m=3)$, and actuators are located at masses $m_{1}, m_{2}, m_{4}$, and $m_{5}(r=4)$. For this scenario, actuators located at $m_{1}$ and $m_{5}$ are essentially isolated from the system by the actuators located at $m_{2}$ and $m_{4}$ (i.e., the system in this configuration has rank 2 , and therefore, only 2 actuators can effectively be placed).

\section{PROBLEM DESCRIPTION}

In recent years there has been a large amount of work done on the problem of actuator placement for the control of flexible structures (Choe and Baruh, 1992; Lim, 1992; Lindberg and Longman, 1984). The fundamental objective behind these studies has been to determine actuator placement configurations for the purposes of vibration suppression. A common strategy adopted in these studies has been to place the actuators at locations where they influence as many modes of the structure as possible to render as many modes of the vibration as controllable as possible. Various methods of quantifying the effectiveness of any given set of actuator locations have been proposed (Choe and Baruh, 1992; Lim, 1992; Lindberg and Longman, 1984).

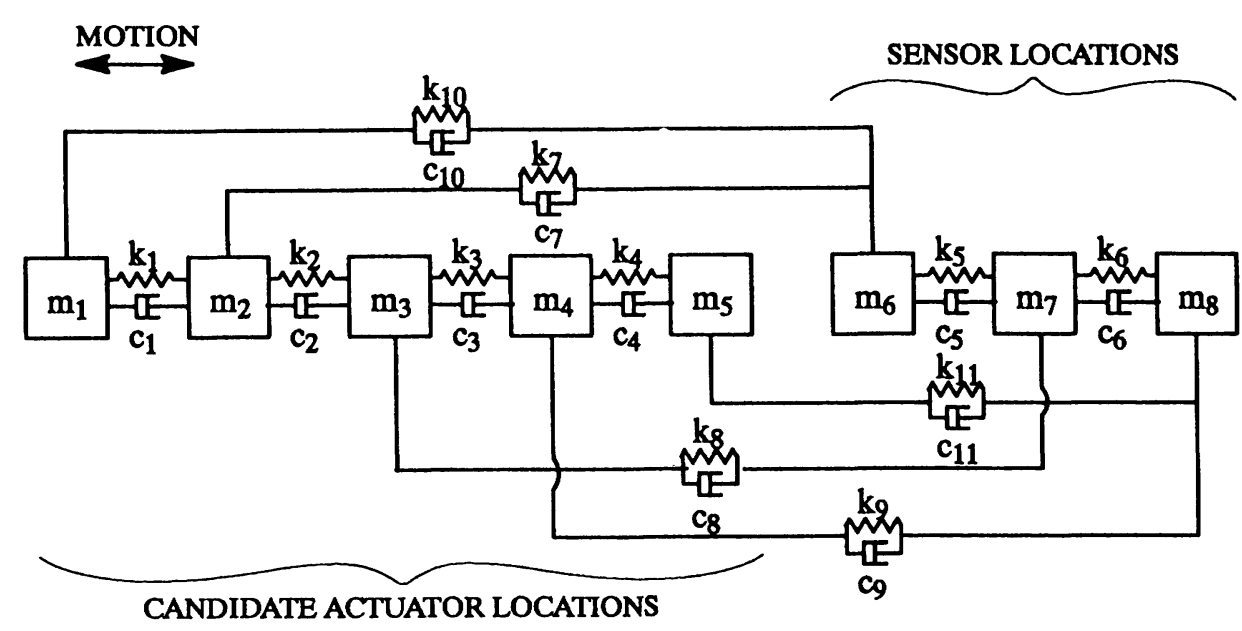

FIGURE 1 8-DOF system. 
The definition of controllability involves the ability to achieve any given state (constant in time) within a finite period of time. Thus actuator configurations that give a high degree of controllability are well suited for vibration suppression problems. However, in the case of a vibration simulator, the interest is not to obtain one given constant state, but rather to obtain a state that varies periodically in time in an arbitrarily specified manner. From this perspective, the problem of actuator placement in vibration simulators includes that of actuator placement for vibration suppression in flexible structures as a special case. Also, because the actuators will typically be operated with displacement commands and will typically have large load capacities, the design constraints will be more on displacement limits rather than force limits.

The following section examines the effectiveness of a given actuator configuration in achieving a specified state of continuous periodic motion through (bounded) periodic excitation.

\section{FORMULATION}

In what follows, it is assumed that rigid body (d.c.) motion can be eliminated by "locking" the actuators. Therefore, any reference to periodic motion implies motion which has no d.c. value because such demands will usually not be made of vibration simulators. It is also assumed that the system has pervasive damping so that any pattern of deformation in a cycle must involve energy dissipation. Both assumptions are valid for the kind of system considered in this article.

The system is assumed to obey a linear model of the form

$$
M \ddot{x}(t)+D \dot{x}(t)+K x(t)=f(t) .
$$

The system model has $m+r+u$ degrees-offreedom (DOF); $m$ DOF represent the motion of the sensor locations (monitored DOF), $r$ DOF represent motion of the actuator locations (excited DOF), and $u$ DOF represent the motion of the remaining unmonitored/unforced DOF of the system (unmonitored DOF).

Let the monitored DOF be arranged in a vector $x_{M}(t)$, the unmonitored DOF in a vector $x_{U}(t)$, and the excited DOF in a vector $x_{R}(t)$. Note, the force vectors corresponding to $x_{M}(t)$ and $x_{U}(t)$ are identically zero. Then, Eq. (1) may be written as

$$
\begin{gathered}
{\left[\begin{array}{c}
M_{M M} M_{M U} M_{M R} \\
M_{U M} M_{U U} M_{U R} \\
M_{R M} M_{R U} M_{R R}
\end{array}\right]\left\{\begin{array}{l}
\ddot{x}_{M} \\
\ddot{x}_{U} \\
\ddot{x}_{R}
\end{array}\right\}} \\
+\left[\begin{array}{c}
D_{M M} D_{M U} D_{M R} \\
D_{U M} D_{U U} D_{U R} \\
D_{R M} D_{R U} D_{R R}
\end{array}\right]\left\{\begin{array}{l}
\dot{x}_{M} \\
\dot{x}_{U} \\
\dot{x}_{R}
\end{array}\right\} \\
+\left[\begin{array}{c}
K_{M M} K_{M U} K_{M R} \\
K_{U M} K_{U U} K_{U R} \\
K_{R M} K_{R U} K_{R R}
\end{array}\right]\left\{\begin{array}{l}
x_{M} \\
x_{U} \\
x_{R}
\end{array}\right\}=\left\{\begin{array}{l}
0 \\
0 \\
f_{R}
\end{array}\right\} .
\end{gathered}
$$

Assuming periodic motion, we write

$$
x(t)=\sum_{k=-N}^{N} X^{(k)} e^{2 \pi i k \omega t},
$$

where $x(t)=\left\{x_{M}, x_{U}, x_{R}\right\}^{T}$. Similarly, we write

$$
f_{R}(t)=\sum_{k=-N}^{N} F_{R}^{(k)} e^{2 \pi i k \omega t}
$$

Using Eqs. (3) and (4), the $k$ th term of Eq. (2) becomes

$$
R^{(k)} X^{(k)}=F^{(k)}
$$

where

$$
R^{(k)}=-k^{2} \omega^{2} M+i k \omega D+K
$$

Keeping in mind that at all times we are referring to a specific $k$ value, we now drop the $k$ superscript for convenience. Thus, at any component frequency within the operational bandwidth, we have

$$
\left[\begin{array}{c}
R_{M M} R_{M U} R_{M R} \\
R_{U M} R_{U U} R_{U R} \\
R_{R M} R_{R U} R_{R R}
\end{array}\right]\left\{\begin{array}{c}
X_{M} \\
X_{U} \\
X_{R}
\end{array}\right\}=\left\{\begin{array}{c}
0 \\
0 \\
F_{R}
\end{array}\right\} .
$$

Displacement and force inputs are considered separately in the following subsections.

\section{Displacement Inputs}

Given a "desired" response, $X_{M}^{d}$, at the sensor locations, the problem is to determine displace- 
ment inputs, $X_{R}$, such that the achieved sensor displacements, $X_{M}$, is as close as possible to $X_{M}^{d}$ in some convenient norm.

We digress for a moment to prove, through physical arguments, that $R_{M M}$ and $R_{U U}$ are nonsingular matrices.

Assume that $X_{R}$ and $X_{U}$ are constrained to have zero displacements (i.e., all DOF, except the monitored DOF, are clamped), then the first equation of Eq. (6) yields

$$
R_{M M} X_{M}=0 .
$$

Because the structure has pervasive damping and there is no energy input to the system (the forced DOF are constrained to have zero displacements), $x_{M}(t)$ cannot sustain a steady periodic motion. Thus, Eq. (7) will not be satisfied for any nonzero $X_{M}$. It follows that $R_{M M}$ must be nonsingular. By similar arguments, it can be shown that $R_{U U}$ is also nonsingular.

Having established that $R_{M M}$ and $R_{U U}$ are invertible, a relationship between $X_{M}$ and $X_{R}$ can be developed as follows. Rewriting the second equation of Eq. (6) as

$$
X_{U}=-R_{U U}^{-1}\left(R_{U M} X_{M}+R_{U R} X_{R}\right),
$$

and combining with the first equation of Eq. (6) results in

$$
\begin{aligned}
& \left(R_{M M}-R_{M U} R_{U U}^{-1} R_{U M}\right) X_{M} \\
& +\left(R_{M R}-R_{M U} R_{U U}^{-1} R_{U R}\right) X_{R}=0 .
\end{aligned}
$$

Again through physical arguments, the coefficient matrix of $X_{M}$ in Eq. (9) must be nonsingular. Therefore,

$$
X_{M}=C_{\mathrm{disp}} X_{R},
$$

where

$$
\begin{aligned}
C_{\text {disp }}=-\left(R_{M M}-R_{M U} R_{U U}^{-1} R_{U M}\right)^{-1} & \left(R_{M R}-R_{M U} R_{U U}^{-1} R_{U R}\right) .
\end{aligned}
$$

\section{Force Inputs}

In general, the actuator subsystem has built in controllers, and inputs to the structure will be in the form of displacement commands to the actuators. However, it might be necessary to take the actuator forces into account, if only to check that very large forces are not required. In such instances, Eq. (8) and the third equation of Eq. (6) can be combined to obtain

$$
\begin{aligned}
\left(R_{R M}-\right. & \left.R_{R U} R_{U U}^{-1} R_{U M}\right) X_{M} \\
& +\left(R_{R R}-R_{R U} R_{U U}^{-1} R_{U R}\right) X_{R}=F_{R} .
\end{aligned}
$$

Using Eq. (10), Eq. (11) can be rewritten as

$$
\begin{aligned}
{\left[\left(R_{R M}-\right.\right.} & \left.R_{R U} R_{U U}^{-1} R_{U M}\right) C_{\mathrm{disp}} \\
& \left.+\left(R_{R R}-R_{R U} R_{U U}^{-1} R_{U R}\right)\right] X_{R}=F_{R} .
\end{aligned}
$$

Equation (13) describes the motion of the system subject only to forces $F_{R}$. When $F_{R}=0$, the system may still exhibit rigid body motion; however, at any nonzero frequency (i.e., nonrigid body modes), due to pervasive damping, steady vibration cannot be sustained. Thus for any nonzero frequency, the only vector $X_{R}$ that satisfies Eq. (13) is the zero vector. It follows that the coefficient of $X_{R}$ in Eq. (13) is nonsingular. Equations (10) and (13) then yield

$$
X_{M}=C_{\text {forc }} F_{R},
$$

where

$$
\begin{aligned}
C_{\text {forc }}= & C_{\mathrm{disp}}\left[\left(R_{R M}-R_{R U} R_{U U}^{-1} R_{U M}\right) C_{\mathrm{disp}}\right. \\
& \left.+\left(R_{R R}-R_{R U} R_{U U}^{-1} R_{U R}\right)\right]^{-1} .
\end{aligned}
$$

We note that because [ $]^{-1}$ in Eq. (15) is of full rank, the columns of $C_{\text {disp }}$ and $C_{\text {forc }}$ span the same subspace of $\mathbf{C}^{m}$. Thus theoretically any motion possible with displacement inputs is also possible with force inputs. However, due to ill conditioning of this matrix, this might not always be true in practice.

\section{Displacement Bounds}

To establish bounds on $X_{M}$, observe that Eqs. (10) and (14) are of the form

$$
X_{M}=P U
$$

where $P$ is a transfer matrix $\left(C_{\text {disp }}\right.$ or $\left.C_{\text {forc }}\right)$ and $U$ is the input $\left(X_{R}\right.$ or $\left.F_{R}\right)$. Using the 2-norm (Golub and Van Loan, 1989), the bounds on $X_{M}$ are as follows:

$$
\left\|X_{M}\right\|_{2}=\|P U\|_{2} \geq \sigma_{\min }\|U\|_{2},
$$

where $\sigma_{\min }$ is the smallest singular value of $P$. Rewriting the above as

$$
\frac{\left\|X_{M}\right\|_{2}}{\sigma_{\min }} \geq\|U\|_{2},
$$


it can be observed that conditions may exist that lead to large input requirements. Conversely, if a bound on the magnitude of $U$ is specified from system limitations, then singular values less than some prescribed tolerance represent outputs that are physically unachievable. For all practical purposes, then, these singular values may be treated as zero, and the transfer matrix may be considered to be numerically rank deficient.

Thus, it is apparent that the conditioning of $P$ could be used as a selection criterion. However, computation of the singular values is quite expensive, rendering this criterion impractical for an exhaustive search of candidate configurations. A criterion based on conditioning should be used only to select from among a small group of "elite" configurations obtained from other selection processes (see the "Best" Configuration below). Alternatively, computationally inexpensive condition number estimators may be used for approximate results (Higham, 1987).

Although we focus on the placement of actuators, we now digress to discuss the unmonitored DOF. These DOF are unmonitored and unforced, and are of indirect interest to the immediate problem. It therefore suffices to show that these DOF are bounded.

From eqs. (8) and (10), $X_{U}$ is expressed in terms of $X_{R}$ as

$$
X_{U}=-R_{U U}^{-1}\left(R_{U M} C_{\mathrm{disp}}+R_{U R}\right) X_{R} .
$$

Therefore,

$$
\left\|X_{U}\right\|_{2} \leq \mid R_{U U}^{-1}\left(R_{U M} C_{\text {disp }}+R_{U R}\right)\left\|_{2}\right\| X_{R} \|_{2},
$$

which is bounded for bounded inputs $X_{R}$.

\section{General Comparison Criterion}

Observe that Eqs. (10) and (14) are of the form $X_{M}=P U$, where $U \in \mathbf{C}^{r}$ is the input (displacement or force), $X_{M} \in \mathbf{C}^{m}$ is the corresponding output, and $P \in \mathbf{C}^{m x r}$ is a transfer matrix ( $C_{\text {disp }}$ or $\left.C_{\text {forc }}\right)$. Because in general $m \neq r, P$ cannot be inverted. In fact, even for the case where $m=r$, $P$ may be rank deficient (see Example 1 below). Therefore, while every input produces a unique output, it is not true that an arbitrary output, $X_{M}^{d}$, should necessarily be achievable through some corresponding input, $U$.

If $\rho=\operatorname{rank}(P)$, then the set of achievable outputs, $X_{M}$, will lie in a $\rho$-dimensional subspace of
$\mathbf{C}^{m}$. Therefore, in the general case, the "best" solution is obtained when $X_{M}$ minimizes the norm of the difference between the desired output, $X_{M}^{d}$, and itself. Via the 2-norm, the best solution is obtained from the simple (but possibly rank deficient) least squares problem,

$$
\min \left\|X_{M}^{d}-X_{M}\right\|_{2} .
$$

Noting that $X_{M}=P U$, the least squares problem becomes

$$
\min \left\|X_{M}^{d}-P U\right\|_{2}
$$

The least squares problem of Eq. (16) can be modified to account for the relative importance of the DOF that comprise the monitored DOF. Let $W$ be a diagonal weighting matrix with positive entries. Then instead of minimizing $\| X_{M}^{d}-$ $P U \|_{2}$, we are now interested in minimizing $\left\|W\left(X_{M}^{d}-P U\right)\right\|_{2}$. Thus the weighting matrix $W$ simply needs to be absorbed into both $X_{M}^{d}$ and the matrix $P$. Without any change in notation, it is henceforth assumed that this has already been done.

Often, in the design stage, bounds on the desired output are not known a priori. However, in cases when these bounds are known a priori, a modification of Eq. (16) is required. Suppose that for each frequency component, bounds of the form

$$
\left|X_{M}^{(i)}\right| \leq a_{i}, i=1,2, \ldots, m
$$

are imposed. These bounds represent a "hypercuboid" in $m$-dimensional space. Without too much loss of accuracy (because these bounds are expected to be approximate), the hypercuboid is approximated by a hyperellipsoid given by

$$
\frac{\left|X_{M}^{(1)}\right|^{2}}{a_{1}^{2}}+\frac{\left|X_{M}^{(2)}\right|^{2}}{a_{2}^{2}}+\cdots+\frac{\left|X_{M}^{(m)}\right|^{2}}{a_{M}^{2}} \leq \beta^{2},
$$

where $\beta$ is some constant.

Equation (18) can then be used to unambiguously compare different actuator configurations based on the worst case value of $\min \left\|X_{M}^{d}-P U\right\|_{2}$ at each component frequency and for each configuration, given that $X_{M}^{d}$ satisfies the given constraint.

Consider matrices $A \in \mathbf{C}^{n \times n}$ and $B \in \mathbf{C}^{n \times m}$, with $n>m$, and assume $B$ is of full rank. Given a unit vector $u$ and a scalar $\mu>0$, let the vector $v$ always be chosen so as to minimize 
$\|A(\mu u)-B v\|_{2}$. If there are no restrictions on the magnitude of $v$, the scalar $\mu$ can be factored out, reducing the problem to that of minimizing $\| A u$ $B v \|_{2}$. We are interested in finding the maximum value that this error norm could take, over all possible choices of the vector $u$.

Let $B=Q R$ be the "thin" $Q R$-decomposition of $B$, where $R \in \mathbf{C}^{m \times m}$, and $Q \in \mathbf{C}^{n \times m}$ satisfies $Q^{H} Q=I$; the superscript $H$ denotes the complex conjugate transpose, and $I$ is the identify matrix. Then, given $u$, we have

$$
v=R^{-1} Q^{H} A u .
$$

Therefore we want to find

$$
\begin{aligned}
\text { error }_{\max } & =\max \left\|A u-B R^{-1} Q^{H} A u\right\|_{2} \\
& =\max \left\|A u-Q Q^{H} A u\right\|_{2},
\end{aligned}
$$

which, by the definition of the 2-norm, is

$$
\text { error }_{\max }=\left\|\left(I-Q Q^{H}\right) A\right\|_{2} .
$$

Now let the singular values of $\left(I-Q Q^{H}\right) A$ be $\sigma_{1} \geq \sigma_{2} \cdots \geq \sigma_{n} \geq 0$. Then error ${ }_{\max }=\sigma_{1}$ for some unit vector $u=u_{1}$, say. However, this is a worst case scenario. For a measure of the overall error, we look next at error ${ }_{\max }$ for all vectors $u$ that are orthonormal to $u_{1}$. This, clearly, is $\sigma_{2}$. Proceeding in this manner and summing the squares of the successive error measures, we get $\sigma_{1}^{2}+\sigma_{2}^{2}+\cdots+\sigma_{n}^{2}$. This is nothing but $\|(I-$ $\left.Q Q^{H}\right) A \|_{F}^{2}$. An overall measure of the error can then be expressed as

$$
\text { error }=\left\|\left(I-Q Q^{H}\right) A\right\|_{F} .
$$

Now let the positive numbers $\beta, a_{1}, a_{2}, \ldots$, $a_{M}$ defined by Eq. (18) be given. Let $A$ be a diagonal matrix with the positive real numbers $a_{i}$ as its diagonal entries. Then for some unit vector $u$ and a real number $\mu>0$, any vector $X_{M}$ may be expresses as $X_{M}=A(\mu u)$. This will automatically satisfy Eq. (18) for $\mu \leq \beta$ and violate it for $\mu>\beta$. Beyond this point, the constant $\mu$ (and hence the constant $\beta$ ) becomes irrelevant.

\section{Algorithm}

At any given frequency, compute the matrix $P$ [ $C_{\text {disp }}$ from Eq. (11) or $C_{\text {forc }}$ from Eq. (15)]. Next, compute its SVD or a rank-revealing QR decomposition or a ULV decomposition. This allows simultaneous evaluation of the conditioning of $P$ and determination of a matrix $Q$ whose columns form an orthonormal basis for the columns of $P$. If $P$ is not well conditioned (numerically rank deficient), the number of columns of the matrix $Q$ should be the same as the numerical rank. Finally, the overall error norm for the given amplitude bounds are evaluated through the Frobenius norm as indicated in Eq. (21).

This process is performed for a number of frequencies in the desired operational bandwidth. Care is taken to select some frequencies close to the relevant resonant frequencies of the system. Finally, compute the 2-norm of the vector of errors (if necessary, a weighted mean square error may be computed). This measure of the error is the general criterion for comparing different actuator configurations.

\section{The Best Configuration}

Use of the criterion developed above may result in several configurations of nearly equal error norm. To choose between these configurations, the conditioning of matrix $P\left[C_{\text {disp }}\right.$ from Eq. (11) or $C_{\text {forc }}$ from Eq. (15)] is utilized. Let $\sigma_{1} \geq$ $\sigma_{2} \cdots \geq \sigma_{\min }$ be the numerically acceptable singular values of $P$, that is, those larger than the tolerance discussed above (see Formulation). Define the ratio $\kappa=\left(\sigma_{1} / \sigma_{\text {min }}\right)_{\text {avg }}$, where the average is over the frequency range (in this article geometric averaging is used). This ratio is a measure of the conditioning of $P$ (hence a measure of the suitability of the actuator configuration), because if $\kappa \gg 1$, then some $X_{M}$ s will require much larger input magnitudes than other $X_{M}$ s. Thus, among all actuator configurations that lead to nearly equal error norm, the one corresponding to the smallest value of $\kappa$ might be considered the best.

In the next section, numerical examples are used to demonstrate the effectiveness of the actuator placement procedure outlined above.

\section{NUMERICAL RESULTS}

The utility of the actuator placement criterion is demonstrated with the eight DOF system shown in Figure 1. The system consists of three sensors and two actuators. The sensor locations are denoted by masses $m_{6}$ through $m_{8}$. Candidate actuator locations are denoted by masses $m_{1}$ through $m_{5}$. For this system, the total number of possible actuator configurations is 10 ; thus it is possible to 
Table 1. System Characteristics for Example 1

$m_{1}=m_{2}=m_{3}=m_{4}=m_{5}=3 ; m_{6}=m_{7}=1.2 ;$
$m_{8}=3.6$
$k_{1}=k_{2}=k_{3}=k_{4}=k_{5}=k_{6}=200 ;$
$k_{7}=k_{8}=k_{9}=100 ; k_{10}=k_{11}=0$
Eigenvalues: 0
$-0.0752 \pm 5.4837 i$
$-0.1260 \pm 7.0977 i$
$-0.2718 \pm 10.4237 i$
$-0.3965 \pm 12.5879 i$
$-0.5654 \pm 15.0277 i$
$-0.6637 \pm 16.2798 i$
$-1.3597 \pm 23.2820 i$

carry out an exhaustive search over the entire set.

The assumption of energy dissipation (pervasive damping) is enforced by the dashpots shown in Figure 1. In both examples discussed below, the mechanical resistance of each dashpot is assumed to be $0.5 \%$ of the associated spring stiffness (i.e., $c_{i}=0.005 k_{i}, i=1,2, \ldots ., 11$ ).

All numerical computations were accomplished with MATLAB (1991). A tolerance of $1 \times 10^{-4}$ was used for the numerical rank calculations of each transfer matrix $P$. For demonstration purposes, this tolerance was chosen sufficiently small so as to affect only a small part of the computed results. However, in practice, we suggest that a larger tolerance $\left(1 \times 10^{-2}\right)$ be used because a value of $1 \times 10^{-4}$ implies that although some output signals may be 10000 times more difficult to produce than others, we still accept them as being achievable.

For each actuator configuration, the frequency range was sampled at 36 equally spaced frequencies and the averaging was done over these frequencies. Uniform frequency weighting was used. It should be noted that in the formulation, uniform spacing and uniform weighting is not a requirement.

Two examples are considered. The first example investigates the effects of output weighting on the placement actuators; it also demonstrates the problem of rank deficiency. The second example investigates the effects of the system's natural frequencies on the placement of actuators.

\section{Example 1}

The system parameters were selected such that mass $m_{3}$ is a nodal point for the first two nonrigid body modes. The resulting system characteristics are shown in Table 1.

A frequency range of 0.1-9 rad/s was considered. This frequency range includes the first two nonzero natural frequencies of the system. Three output weighting scenarios were considered: $A=$ $\operatorname{diag}[1,1,1], A=\operatorname{diag}[1,10,1]$, and $A=\operatorname{diag}$ $[10,1,10]$. Note that the first weighting matrix is simply the identity matrix and corresponds to the situation where a priori knowledge is not available and therefore it is assumed that all outputs are equally significant.

The results are given in Tables 2 and 3. The first table corresponds to displacement input considerations and the second table to force input considerations. As mentioned earlier, from physical considerations we might expect that any output achievable through displacement inputs is also achievable through force inputs. This is indeed true in exact arithmetic. However, it may happen that a small amplitude of vibration near a

Table 2. Displacement Comparison for Example 1

\begin{tabular}{|c|c|c|c|c|c|c|c|c|}
\hline \multicolumn{3}{|c|}{$A=\operatorname{diag}[1,1,1]$} & \multicolumn{3}{|c|}{$A=\operatorname{diag}[1,10,1]$} & \multicolumn{3}{|c|}{$A=\operatorname{diag}[10,1,10]$} \\
\hline Error & $\kappa$ & Location & Error & $\kappa$ & Location & Error & $\kappa$ & Location \\
\hline 6.00 & 5.74 & 2,4 & 46.03 & 6.80 & 3,4 & 33.68 & 6.20 & 2,3 \\
\hline 6.00 & 6.20 & 2,3 & 46.03 & 14.88 & 3,5 & 33.68 & 14.46 & 1,3 \\
\hline 6.00 & 6.80 & 3,4 & 49.56 & 5.74 & 2,4 & 34.35 & 5.74 & 2,4 \\
\hline 6.00 & 8.83 & 2,5 & 49.56 & 8.83 & 2,5 & 34.35 & 8.83 & 2,5 \\
\hline 6.00 & 9.79 & 1,4 & 49.56 & 9.79 & 1,4 & 34.35 & 9.79 & 1,4 \\
\hline 6.00 & 10.16 & 1,5 & 49.56 & 10.16 & 1,5 & 34.35 & 10.16 & 1,5 \\
\hline 6.00 & 14.46 & 1,3 & 50.02 & 6.20 & 2,3 & 38.95 & 6.80 & 3,4 \\
\hline 6.00 & 14.88 & 3,5 & 50.02 & 14.46 & 1,3 & 38.95 & 14.88 & 3,5 \\
\hline 8.49 & 1.00 & 1,2 & 51.52 & 1.00 & 4,5 & 66.84 & 1.00 & 1,2 \\
\hline 8.49 & 1.00 & 4,5 & 52.96 & 1.00 & 1,2 & 67.96 & 1.00 & 4,5 \\
\hline
\end{tabular}


Table 3. Force Comparison for Example 1

\begin{tabular}{|c|c|c|c|c|c|c|c|c|}
\hline \multicolumn{3}{|c|}{$A=\operatorname{diag}[1,1,1]$} & \multicolumn{3}{|c|}{$A=\operatorname{diag}[1,10,1]$} & \multicolumn{3}{|c|}{$A=\operatorname{diag}[10,1,10]$} \\
\hline Error & $\kappa$ & Location & Error & $\kappa$ & Location & Error & $\kappa$ & Location \\
\hline 6.00 & 9.80 & 2,4 & 46.10 & 16.67 & 3,4 & 34.35 & 9.81 & 2,4 \\
\hline 6.00 & 9.80 & 1,5 & 46.10 & 21.24 & 3,5 & 34.35 & 9.81 & 1,5 \\
\hline 6.00 & 13.27 & 1,4 & 49.56 & 9.81 & 2,4 & 34.35 & 13.27 & 1,4 \\
\hline 6.00 & 16.85 & 2,5 & 49.56 & 9.81 & 1,5 & 34.35 & 16.85 & 2,5 \\
\hline 6.08 & 15.94 & 2,3 & 49.56 & 13.27 & 1,4 & 35.06 & 15.94 & 2,3 \\
\hline 6.08 & 16.67 & 3,4 & 49.56 & 16.85 & 2,5 & 35.06 & 18.56 & 1,3 \\
\hline 6.08 & 18.56 & 1,3 & 50.08 & 15.94 & 2,3 & 40.15 & 16.67 & 3,4 \\
\hline 6.08 & 21.24 & 3,5 & 50.08 & 18.56 & 1,3 & 40.15 & 21.24 & 3,5 \\
\hline 8.49 & 1.00 & 1,2 & 51.52 & 1.00 & 4,5 & 66.84 & 1.00 & 1,2 \\
\hline 8.49 & 1.00 & 4,5 & 52.96 & 1.00 & 1,2 & 67.96 & 1.00 & 4,5 \\
\hline
\end{tabular}

resonant frequency, imparted at a point very close to a nodal point of the corresponding eigenvector, may lead mathematically to some desired response. Yet the same amplitude of vibration may require a very large force input, making it unachievable. This can lead to discrepancies between displacement (based and force) based calculations; such discrepancies will become noticeable when the tolerance used in the computation of the rank of $P$ is chosen sufficiently large.

As seen from Tables 2 and 3, the computed errors corresponding to more than one actuator location is the same. In those instances, the conditioning of the system, $\kappa$, was used to select the best from among these configurations. However, in these examples, the number of candidate configurations was sufficiently small, allowing computation of the conditioning for all configurations. The conditioning was then used to order the configurations as shown in Tables 2 and 3.

Note that the conditioning, $\kappa$, for the two lowest ranked configurations is exactly 1 . These two configurations correspond to rank-deficient cases (rank $=1$ ); in these cases, $\sigma_{1}=\sigma_{\min }$, thus the conditioning evaluated from $\kappa=\sigma_{1} / \sigma_{\min }$, is iden-

Table 4. System Characteristics for Example 2

$$
\begin{gathered}
m_{1}=m_{2}=m_{3}=m_{4}=m_{5}=m_{6}=m_{7}=2 ; m_{8}=0.6 \\
k_{1}=k_{2}=k_{3}=k_{4}=220 ; k_{5}=k_{6}=200 ; \\
k_{7}=k_{8}=k_{9}=k_{10}=k_{11}=125
\end{gathered}
$$

Eigenvalues: 0

$$
\begin{aligned}
& -0.1595 \pm 0.4837 i \\
& -0.4217 \pm 12.9807 i \\
& -0.5170 \pm 14.3711 i \\
& -0.8074 \pm 17.9528 i \\
& -0.8933 \pm 16.8816 i \\
& -1.1570 \pm 21.4816 i \\
& -2.1191 \pm 29.0372 i
\end{aligned}
$$

tically 1 . In the next example, the rank deficiency disappears due to the coupling from springs $k_{10}$ and $k_{11}$.

It appears that for the scenarios considered in this example, the overall best actuator placement is the 2,4 configuration (i.e., actuators placed at $m_{2}$ and $m_{4}$ ).

\section{Example 2}

The system characteristics for this are shown in Table 4. Again the system parameters were chosen such that mass $m_{3}$ is a nodal point for the first two nonrigid body modes.

\begin{tabular}{|c|c|c|c|c|c|}
\hline \multicolumn{6}{|c|}{$A=\operatorname{diag}[1,10,1]$} \\
\hline \multicolumn{3}{|c|}{ Frequency: 4.0-9.0 } & \multicolumn{3}{|c|}{ Frequency: $4.0-13.1$} \\
\hline Error & $\kappa$ & Location & Error & $\kappa$ & Location \\
\hline 45.00 & 8.93 & 1,5 & 40.10 & 4.63 & 1,5 \\
\hline 46.12 & 7.22 & 1,4 & 42.37 & 4.67 & 2,5 \\
\hline 46.35 & 7.58 & 2,5 & 43.10 & 4.76 & 1,4 \\
\hline 47.09 & 5.00 & 2,4 & 44.67 & 3.81 & 2,4 \\
\hline 50.18 & 4.68 & 2,3 & 45.97 & 5.95 & 2,3 \\
\hline 50.18 & 6.80 & 1,3 & 45.97 & 7.04 & 1,3 \\
\hline 50.18 & 8.06 & 1,2 & 45.97 & 9.60 & 1,2 \\
\hline 51.37 & 4.87 & 3,4 & 47.34 & 5.71 & 3,4 \\
\hline 51.37 & 7.23 & 3,5 & 47.34 & 5.99 & 3,5 \\
\hline 51.37 & 7.41 & 4,5 & 47.34 & 6.88 & 4,5 \\
\hline
\end{tabular}

In this example two frequency ranges were considered but the output weighting was fixed at $A=\operatorname{diag}[1,10,1]$. The first frequency range, 4.0 $9.0 \mathrm{rad} / \mathrm{s}$, avoids the system natural frequencies. The second frequency range, $4.0-13.1 \mathrm{rad} / \mathrm{s}$, includes the system's second natural frequency. Comparisons for displacement inputs and force inputs are shown in Tables 5 and 6, respectively.

Table 5. Displacement Comparison for Example 2 
Table 6. Force Comparison for Example 2

\begin{tabular}{|c|c|c|c|c|c|}
\hline \multicolumn{6}{|c|}{$A=\operatorname{diag}[1,10,1]$} \\
\hline \multicolumn{3}{|c|}{ Frequency: 4.0-9.0 } & \multicolumn{3}{|c|}{ Frequency: $4.0-13.1$} \\
\hline Error & $\kappa$ & Location & Error & $\kappa$ & Location \\
\hline 45.00 & 2.82 & 1,5 & 40.70 & 2.58 & 1,5 \\
\hline 46.12 & 2.81 & 1,4 & 42.37 & 2.51 & 2,5 \\
\hline 46.35 & 2.95 & 2,5 & 43.10 & 2.94 & 1,4 \\
\hline 47.09 & 2.73 & 2,4 & 44.67 & 2.63 & 2,4 \\
\hline 50.18 & 4.05 & 2,3 & 45.97 & 4.01 & 2,3 \\
\hline 50.18 & 4.18 & 1,3 & 45.97 & 4.26 & 1,3 \\
\hline 50.18 & 17.25 & 1,2 & 45.97 & 15.93 & 1,2 \\
\hline 51.37 & 4.92 & 3,5 & 47.34 & 3.85 & 3,5 \\
\hline 51.37 & 5.05 & 3,4 & 47.34 & 3.96 & 3,4 \\
\hline 51.37 & 20.43 & 4,5 & 47.34 & 14.35 & 4,5 \\
\hline
\end{tabular}

Tables 5 and 6 indicate that the ranking of the configurations are identical except for second and third ranked elements that are sometimes reversed. Thus, it appears that system frequencies in the operational bandwidth does not drastically affect the placement of the actuators. However, note that both the maximum error and the system conditioning are substantially smaller for the cases involving system frequencies in the operational bandwidth.

\section{CONCLUSIONS}

A criterion for selecting actuator placement in MDOF vibration simulators has been presented. The criterion incorporates not only information regarding the locations of the sensors, but also the relative importance assigned to different sensor outputs and any a priori information regarding amplitude bounds at different sensor locations. The selection criterion makes it possible to unambiguously compare different actuator configurations.

The numerical results presented in this article indicate that actuator placement in MDOF vibration simulators is independent of nodal locations and system frequencies. This is quite surprising considering the fact that in vibration suppression problems, nodal locations and system frequencies are very critical to the placement of actuators. From these numerical results, it appears that no simple rule of thumb criterion exists for the placement of actuators in MDOF vibration simulator systems.
In real systems, the number of candidate actuator locations will be much larger than the 10 used in the examples presented here; therefore, an exhaustive search based primarily on the selection criterion of this study will be impractical. However, through engineering judgement and physical constraints, the set of candidate configurations can be reduced to a manageable size before the selection criterion is applied. Thus, the selection criterion presented in this article is intended to compliment the current use of engineering judgement and physical constraints in the design of MDOF vibration simulators.

This research was supported by US Army contract DAAH03-92-P-0893 through the Dynamic Test Branch of Redstone Technical Test Center.

\section{REFERENCES}

Choe, K., and Baruh, H., 1992, "Actuator Placement in Structural Control,' Journal of Guidance, Control, and Dynamics, Vol. 15, pp. .40-47.

Fitz-Coy, N. G., 1991, "Dynamic Analysis of the 'Road Simulator' Motion Simulator,' Final Report submitted to the US Army Research Office.

Golub, G. H., and Van Loan, C. F., 1989, Matrix Computations, 2nd Ed., Johns Hopkins University Press, Baltimore, MD.

Hahn, H., and Raasch, W., 1986, "Multi-Axis Vibration Test on Spacecraft Using Hydraulic Exciters," AGARD Mechanical Qualification of Large Space Structures, 23.1-23.23.

Higham, N. J., 1987, "A Survey of Condition Number Estimation for Triangular Matrices," SIAM Review, Vol. 29, pp. 575-596.

Lim, K. B., 1992, "Method for Optimal Actuator and Sensor Placement for Large Flexible Structures," Journal of Guidance, Control, and Dynamics, Vol. 15, pp. 49-57.

Lindberg, R. E., Jr., and Longman, R. W., 1984, “'On the Number and Placement of Actuators for Independent Modal Space Control,' Journal of Guidance, Control, and Dynamics, Vol. 7, pp. 215-221.

MATLAB User's Guide, 1991, The Math Works, South Natick, MA.

Raasch, W., 1983, "Hydraulic Actuators and Associated Components Suited for Multi-Axis Vibration Systems," ESTEC.

Schmidt, A., 1983, "Control of Multi-Axis Vibration Systems," ESTEC.

Woyski, W., and Tauscher, W., 1992, "Multiple Degree of Freedom Motion Simulation," Paper presented at the Spacecraft Dynamics Environment Technical Interchange Meeting. 

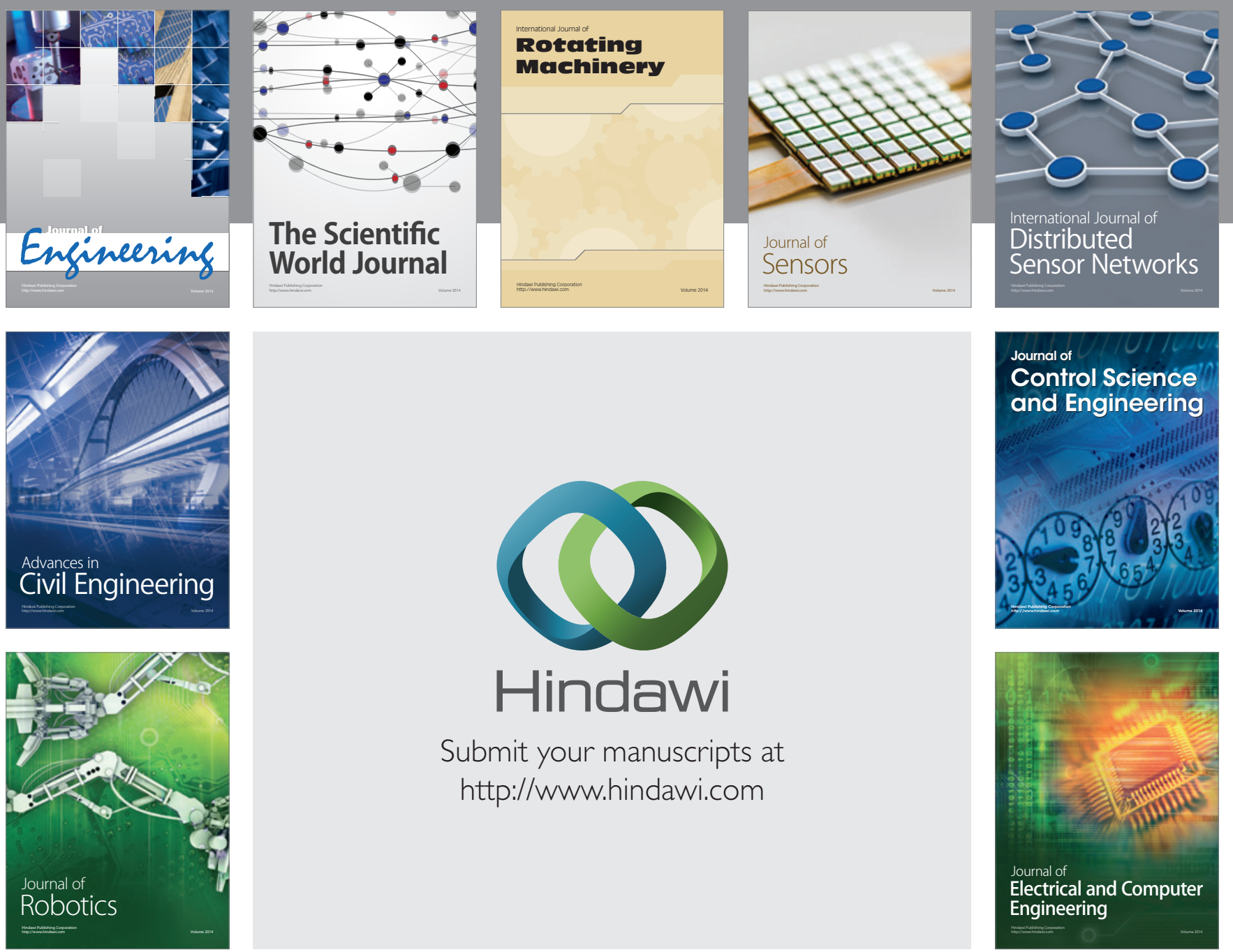

Submit your manuscripts at

http://www.hindawi.com
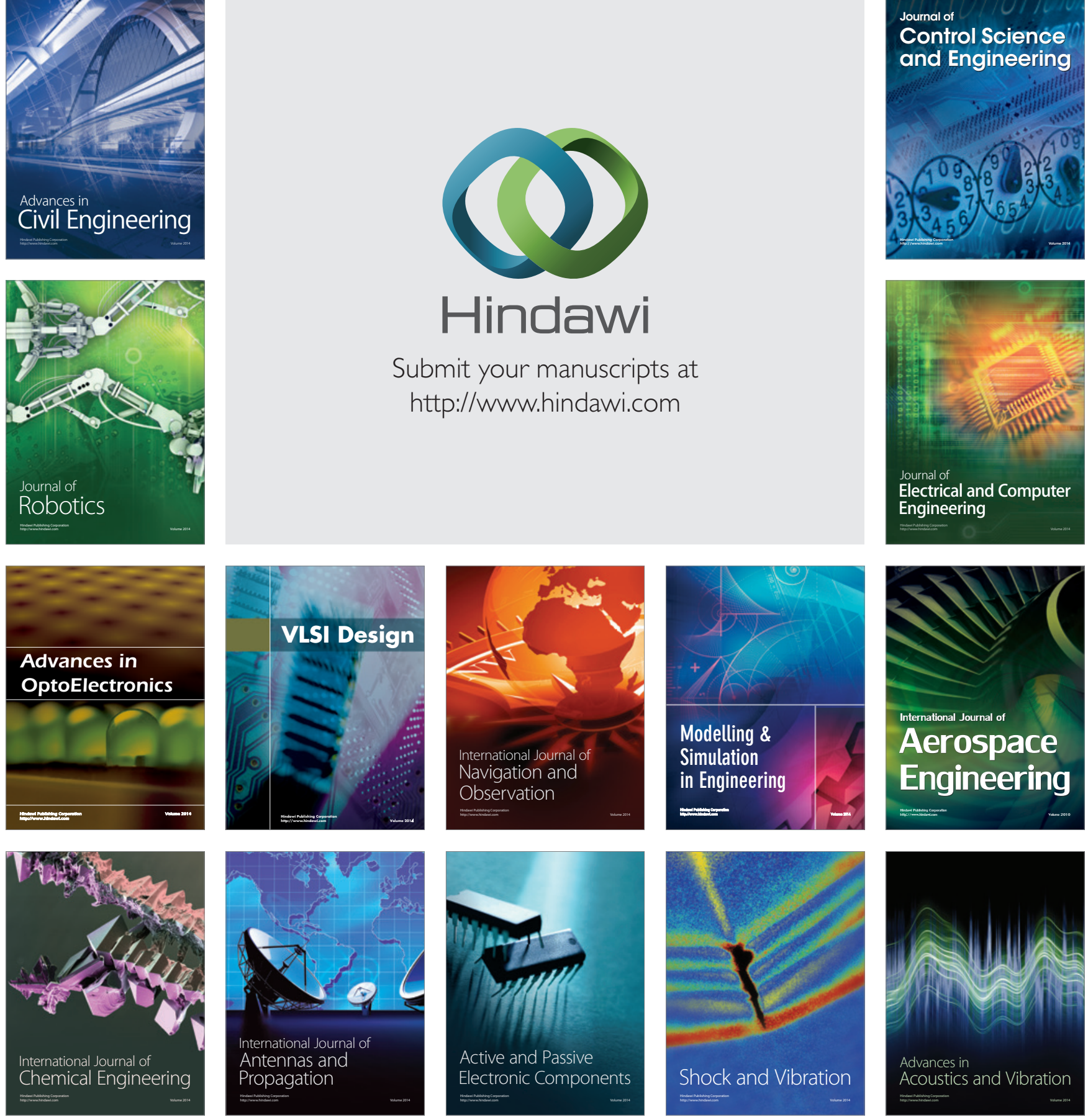